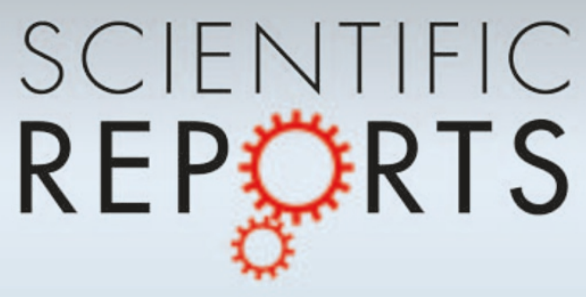

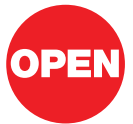

SUBJECT AREAS:

PLANT SCIENCES

PLANT CELL BIOLOGY

PLANT BIOTECHNOLOGY

MOLECULAR ENGINEERING

Received

29 September 2011

Accepted

12 March 2012

Published

27 March 2012

Correspondence and requests for materials should be addressed to P.P. (p.perata@sssup.

\section{Metabolic engineering of the iodine content in Arabidopsis}

\author{
Martina Landini' ', Silvia Gonzali' ', Claudia Kiferle', Massimo Tonacchera ${ }^{2,4}$, Patrizia Agretti ${ }^{2,4}$, \\ Antonio Dimida ${ }^{2,4}$, Paolo Vitti ${ }^{2,4}$, Amedeo Alpi ${ }^{3,4}$, Aldo Pinchera ${ }^{2,4}$ \& Pierdomenico Perata ${ }^{1}$
}

\begin{abstract}
'PlantLab, Institute of Life Sciences, Scuola Superiore Sant'Anna, Piazza Martiri della Libertà 33, 56127 Pisa, Italy, ${ }^{2}$ Department of Endocrinology and Metabolism, University of Pisa, Via Paradisa 2, 56124 Pisa, Italy, ${ }^{3}$ Department of Crop Plant Biology, University of Pisa, Via Mariscoglio 34, 56124 Pisa, Italy, ${ }^{4}$ Centre of Excellence for the study of damage to the Nervous and Endocrine systems produced by Environmental, Alimentary, and Pharmacological agents, AmbiSEN, University of Pisa.
\end{abstract}

Plants are a poor source of iodine, an essential micronutrient for human health. Several attempts of iodine biofortification of crops have been carried out, but the scarce knowledge on the physiology of iodine in plants makes results often contradictory and not generalizable. In this work, we used a molecular approach to investigate how the ability of a plant to accumulate iodine can be influenced by different mechanisms. In particular, we demonstrated that the iodine content in Arabidopsis thaliana can be increased either by facilitating its uptake with the overexpression of the human sodium-iodide symporter (NIS) or through the reduction of its volatilization by knocking-out HOL-1, a halide methyltransferase. Our experiments show that the iodine content in plants results from a balance between intake and retention. A correct manipulation of this mechanism could improve iodine biofortification of crops and prevent the release of the ozone layer-threatening methyl iodide into the atmosphere.

odine is an essential micronutrient for human health, as it is required for the synthesis of the thyroid hormones: thyroxine and tri-iodothyronine ${ }^{1}$. The sodium-iodide symporter (NIS) drives uptake of iodide across the basolateral membrane of the thyrocytes. NIS is also present in other tissues where iodine is concentrated, such as gastric mucosa, salivary glands, and lactating mammary glands ${ }^{1}$. Iodine deficiency affects about two billion people worldwide, and results in a series of disorders including goitre and mental retardation ${ }^{2}$. Iodization of salt is the most common method for dietary iodine supplementation ${ }^{3}$. However, iodine salts may be volatile, and losses may occur during transport, storage and cooking ${ }^{2}$.

Biofortification of crops with iodine would represent a cost-effective way to prevent its deficiency, since iodine in foodstuffs is readily bioavailable and assimilated ${ }^{4,5}$. Plants can accumulate this element, but fruits and vegetables are in general poor sources of it. Increasing iodine applications to the soil can result in an enhanced iodine accumulation in crops ${ }^{4,6}$. However, results are often inconsistent and dependent on the plant species, the iodine source and the methods of feeding, indicating that a better understanding of the factors affecting the ability of a plant to accumulate iodine would be necessary to set up reliable biofortification programs.

The physiology of iodine in plant is poorly characterized. Plants can absorb iodine from the soil and it is assumed that in root cells iodine fluxes across membranes through putative $\mathrm{H}^{+}$/halide transporters and anion channels ${ }^{7}$. However, the molecular identities of such transporters have not yet been established ${ }^{7,8}$. Once inside the plant, a xylematic flux of iodine seems to be predominant ${ }^{4,6,9,10}$, although a phloematic route for this element has been recently observed in tomato ${ }^{11}$. Methyl iodide emission from the plant aboveground organs has been recently described in different species ${ }^{12-15}$. Responsible for this iodine volatilization are specific enzymes possessing a Sadenosyl-L-methionine -dependent methyltransferase activity ${ }^{13-15}$. Particular attention has to be paid to this phenomenon since it contributes to the general mechanism of methyl halide emissions from natural sources, threatening the stratospheric ozone layer ${ }^{13,14}$.

Metabolic engineering has been successful in fortifying crops with several nutrients ${ }^{16}$, including folate ${ }^{17}$ and iron ${ }^{18}$ but, to date, no reports of enhancement of iodine content of plants using a molecular approach have been published. In this work, we describe how two different and opposite mechanisms, intake and release, can modulate the dynamics of iodine in the model plant Arabidopsis thaliana. 
a

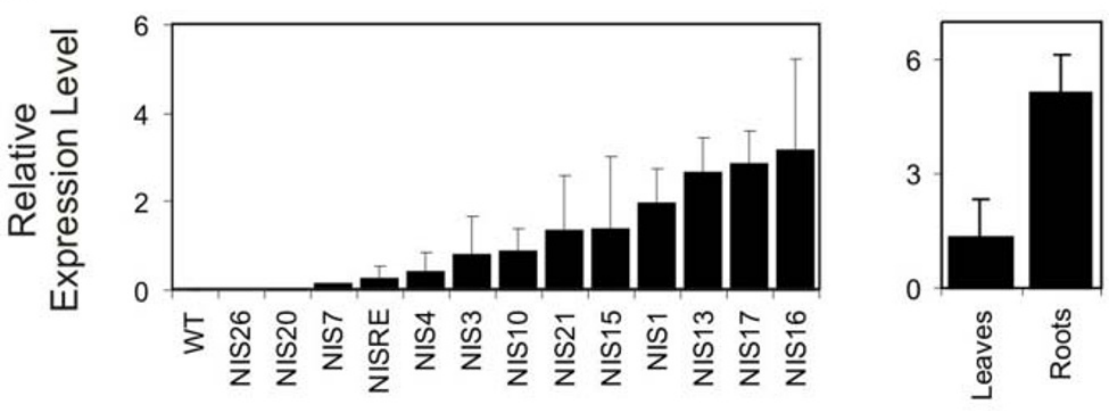

$\mathrm{b}$
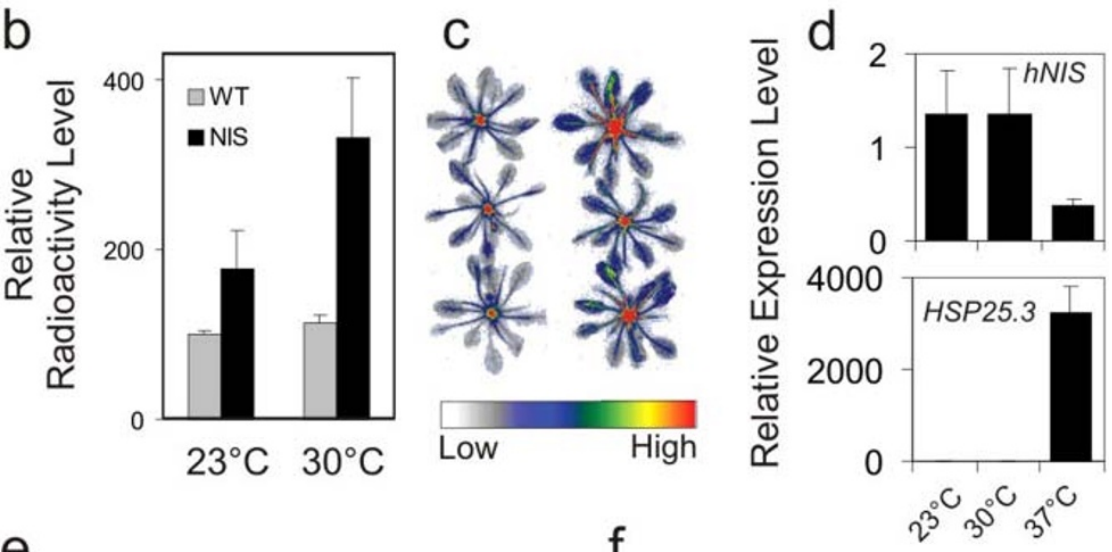

e
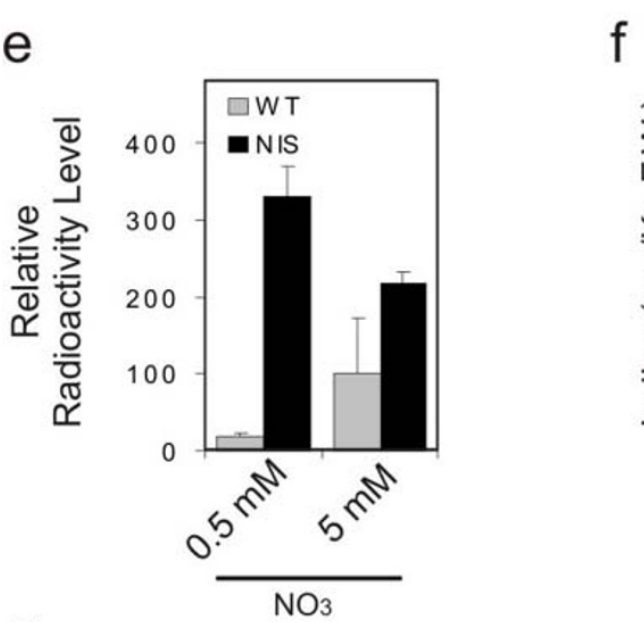

f
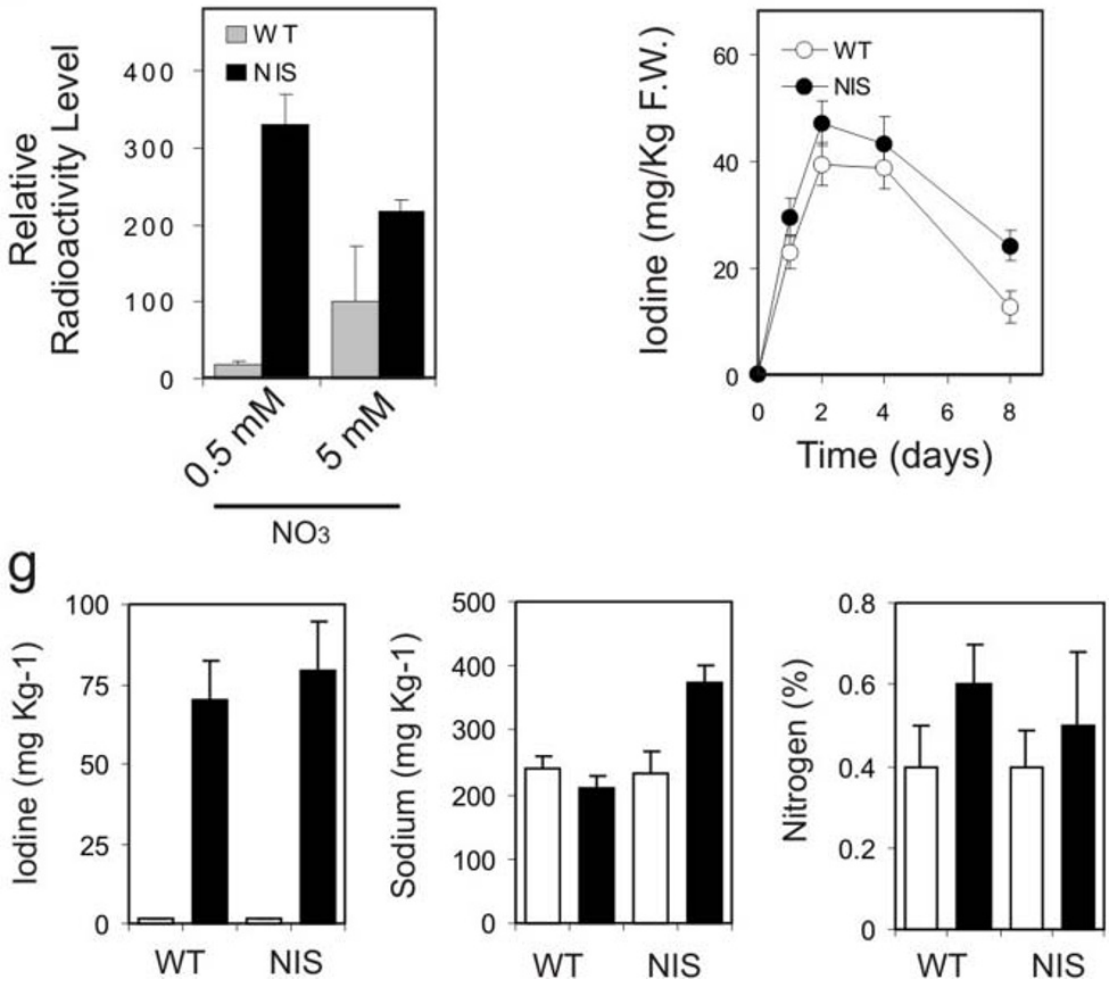

Figure 1 Expression of human NIS in Arabidopsis plants. (a) Selection of transgenic Arabidopsis lines expressing different levels of NIS. Expression of NIS was measured by qRT-PCR ( $\mathrm{n}=3$, \pm s.d., expression in NIS10 $=1)$; expression in leaves and roots from line 16 is also shown. (b) Uptake of ${ }^{125}$ I by wild-type (WT) and NIS plants (NIS) at different temperatures ( $\mathrm{n}=3, \pm$ s.d.). (c) Organ-distribution of ${ }^{125} \mathrm{I}$ in WT and over-expressors of NIS kept at $30^{\circ} \mathrm{C}$. A color scale, indicating the different levels of radioactivity, is shown. (d) Effect of temperature on the expression of $h N I S$ in NIS plants (Line 16). The expression of HSP25.3 is also shown as a control for the heat response of plants. Expression of $h N I S$ and $H S P 25.3$ was measured by qRT-PCR ( $\mathrm{n}=3$, \pm s.d.). Expression at $23^{\circ} \mathrm{C}$ in one of the replicates was taken as a reference and its value set at 1 . (e) Effect of different nitrate concentrations on ${ }^{125} \mathrm{I}$ uptake in WT and NIS plants ( $\mathrm{n}=3, \pm$ s.d.). (f) Iodine content after feeding $35 \mu \mathrm{mol}$ non-radioactive iodide in WT and over-expressors of NIS. In this experiment, plants were grown in soil and KI was used as a source of iodine. Comparable results were obtained by either growing plants in a hydroponic system (data not shown) or giving NaI as a source of iodine. (g) Iodine, sodium and total nitrogen content in WT and NIS plants grown in a hydroponic system without (white bars) or with $30 \mu \mathrm{M} \mathrm{NaI}$ (black bars) for four weeks $(\mathrm{n}=3)$. 


\section{Results}

Since plant root transporters for iodine have not yet been isolated, we attempted to increase the iodine uptake of Arabidopsis thaliana plants by expressing the human sodium-iodide symporter (hNIS) protein under the control of the CaMV 35 S promoter. Several independent transgenic lines were obtained with different levels of expression of the $h N I S$ gene (Fig 1a). Line 16 was used for the experiments described in this paper, which were confirmed also by using line 17 (not shown). In NIS plants, $h N I S$ expression was higher in roots than in leaves (Fig 1a). Radioactive ${ }^{125} \mathrm{I}$ (approximately $0.3 \mathrm{pmol}$ ) were fed to wild type (WT) and NIS transgenic plants, which were kept at different temperatures. NIS plants accumulated more radioactive iodine, and this was particularly evident when plants were fed iodine at $30^{\circ} \mathrm{C}$ (Fig. $1 \mathrm{~b}$ ), a temperature that does not enhance the expression of $h N I S$ (Fig. 1d) but is closer to the physiological environment of the human thyroid. At $37^{\circ} \mathrm{C}$ the plants suffered from heat stress, as demonstrated by the induction of HSP25.3, and this reduced the expression of $h N I S$ (Fig. 1d). Iodine accumulated at a higher level in the young parts of the plant (Fig. 1c). Nitrate is an essential nutrient for plants, but can negatively affect the activity of NIS ${ }^{19}$. Increasing the nitrate level had a positive impact on the ${ }^{125} \mathrm{I}$ uptake in WT plants, but decreased the iodine content in NIS plants (Fig. 1e), in line with its negative effect on the NIS transporter ${ }^{19}$. Because of the short duration of the treatment with high temperature or low nitrate, which were both limited to the iodine administration (approx. a week), no negative effects on plant growth were observed (data not shown). No differences related to the genotype were observed in the content of total nitrogen of the plants (Fig. 1g), indicating that the activity of NIS did not interfere with nitrogen metabolism. A positive effect of iodine feeding on nitrogen content was observed (Fig. 1g) and, although not relevant in the context of iodine biofortification, would be worthy of further study.

These results suggested that the presence of NIS enabled Arabidopsis plants to accumulate more iodine. However, when we tried to feed plants with non-radioactive iodide, applied at a quantity more appropriate for a biofortification approach $(35 \mu \mathrm{mol})$, we were puzzled by the comparable final iodine contents in WT and NIS plants (Fig. 1f). Remarkably, while iodine content in NIS plants did not differ from that of the wild-type, the content of sodium increased in NIS plants, indicating that the NIS protein was active in the transgenic plants (Fig. 1g). The NIS protein act as a symporter of sodium and iodide and the increased sodium content observed in the NIS plants would have predicted an increased iodine content too, which was instead not observed (Fig. $1 \mathrm{f}, \mathrm{g}$ ). As the difference in the two types of experiments (radioactive vs. non-radioactive iodide) depends on the iodine concentration used, we hypothesized that, in addition to iodine uptake, iodine retention could also play an important role, affecting the final iodine level measured in plants. High levels of iodine can be toxic to plants ${ }^{20}$, and they may possess a homeostatic mechanism to get rid of excessive iodine.

An important player in iodine removal from plant tissues in Arabidopsis could be HOL-1, a methyltransferase that catalyzes the S-adenosyl-L-methionine-dependent methylation of halides, including iodide, to produce volatile methyl iodide ${ }^{13}$. We therefore analyzed a hol-1 mutant line known to be unable to produce methyl iodide ${ }^{13}$ and found it displayed a significantly higher ${ }^{125}$ I content than WT, when plants were fed 0.3 pmol radioactive ${ }^{125} \mathrm{I}$ (Fig. 2a). Again, most of the ${ }^{125} \mathrm{I}$ accumulated in the apex and young leaves (Fig. 2b). Not only the quantity, but also the distribution of iodine was wider than WT in hol-1 plants, as indicated by the translocation of ${ }^{125} \mathrm{I}$ up to the inflorescence tip (Fig. 2c). When $35 \mu \mathrm{mol}$ non-radioactive iodide was fed, hol-1 plants accumulated much higher levels of iodine compared to WT (Fig. 2d), without any negative impact on biomass at the iodine amount fed to the plants (not shown). Additional experiments on the impact of iodine on plant growth parameters showed that only after repeated feeding with iodine a reduction in growth can be observed, which was however largely independent of the genotype a

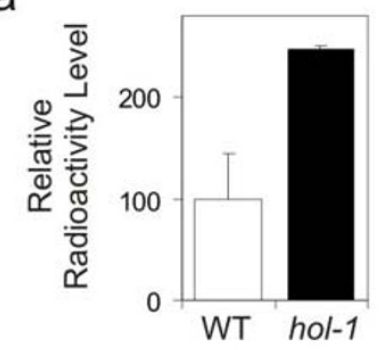

d

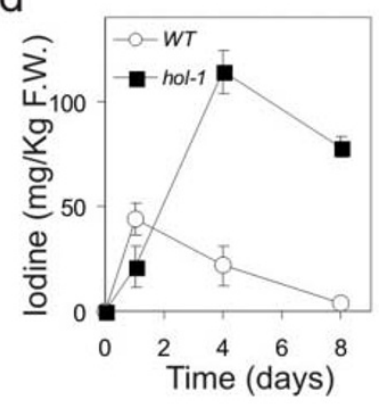

b

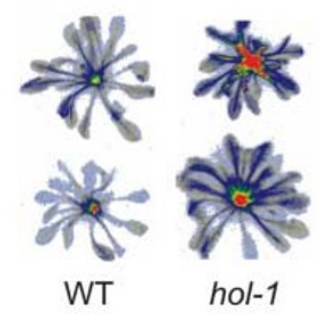

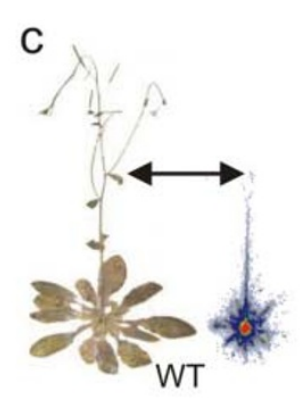

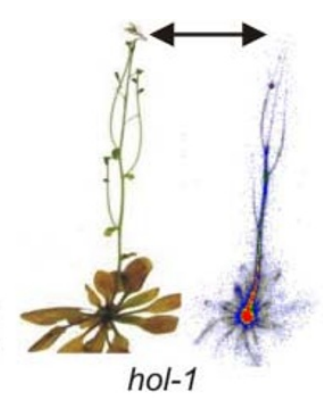

hol-1
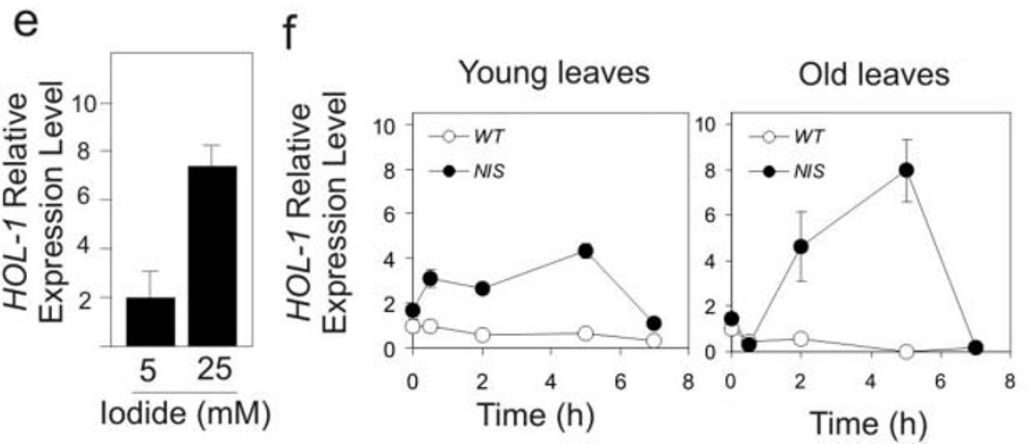

Figure $2 \mid$ Impact of HOL-1 on iodine dynamics in plants. (a) ${ }^{125} \mathrm{I}$ uptake in Arabidopsis wild type (WT) plants and in mutants lacking HOL-1 activity (hol-1) $\left(\mathrm{n}=3\right.$, \pm s.d.). Distribution of ${ }^{125}$ I radioactivity in WT and hol-1 plants at the rosette stage (b) and at the flowering stage (c). Iodine treatments were carried out at $23^{\circ} \mathrm{C}$ as described in Figure 1. Color scale is shown in Figure 1c. (d) Iodine content in WT and in hol-1 plants after feeding $35 \mu \mathrm{mol}$ nonradioactive iodide. (e) Induction of $H O L-1$ expression by iodide. 14d-old seedlings grown in liquid culture were fed iodide at the concentrations shown. The expression of HOL- 1 was measured at time 0 and $20 \mathrm{~min}$. after the treatment by qRT-PCR $(\mathrm{n}=3$, \pm s.d., value at time $0=1$ ). (f) $H O L-1$ gene expression in WT and over-expressors of NIS (NIS) fed with $25 \mathrm{mM}$ non-radioactive iodide. Expression was measured by qRT-PCR ( $\mathrm{n}=3$, \pm s.d., value in WT at time $0=1$ ). 
a
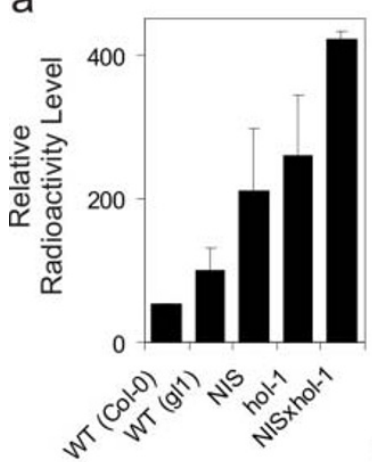

C

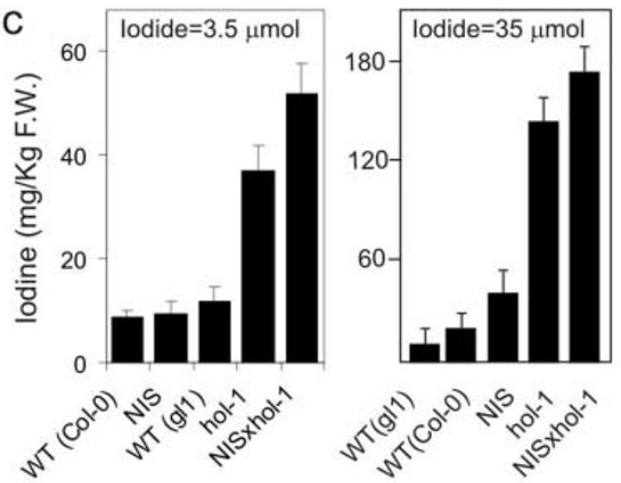

Figure 3 Enhanced iodine uptake in Arabidopsis plants overexpressing the human NIS in the hol-1 background. (a) ${ }^{125}$ I uptake in Arabidopsis plants lacking HOL-1 (hol-1) and over-expressing hNIS (NISxhol-1), in comparison to wild type (WT), NIS and hol-1 plants ( $\mathrm{n}=3$, \pm s.d.). (b) Organ-distribution of ${ }^{125} \mathrm{I}$ in WT, NIS, hol-1 and NISxhol-1 flowering plants. Iodine treatments were carried out at a $\mathrm{T}^{\circ}=30^{\circ} \mathrm{C}$. Color scale is shown in Figure 1c. (c) Iodine content ( $\mathrm{n}=3$, \pm s.d.) in WT, NIS, hol-1 and NISxhol-1 plants after feeding 3.5 and $35 \mu \mathrm{mol}$ non-radioactive iodide $\left(\mathrm{T}^{\circ}=30^{\circ} \mathrm{C}\right)$. In this set of experiments, two different wild type genotypes ( Col-0 and gll) were tested, representing the genetic backgrounds of hol-1 and NIS plants, respectively.

(Fig. S1). The levels of iodine in hol-1 plants decreased four days after feeding, demonstrating that, besides HOL-1, there must be a second metabolism for removing the excess iodine from the plant, possibly involving other HOL methyltransferases ${ }^{13,14}$.

$H O L-1$ is expressed constitutively at relatively high levels in Arabidopsis plants (Fig. S2). Interestingly, HOL-1 expression resulted to be further induced by iodide (Fig. 2e) and in NIS plants fed with iodine a higher and prolonged induction of this gene was observed over the time, particularly in the older leaves (Fig. 2f).

We finally crossed NIS plants with the hol-1 mutant and found that ${ }^{125}$ I feeding resulted in iodine content in NISxhol-1 $>$ hol-1 $>$ NIS $>W T$ (Fig. 3a). Translocation of ${ }^{125} \mathrm{I}$ along the inflorescence was very efficient in NIS, hol-1 and NISxhol-1 plants (Fig. 3b). Following iodide administration at micromolar levels, iodine content was effectively enhanced in NISxhol-1 plants compared to both the parental lines, although with a mild difference with respect to hol-1 plants.

\section{Discussion}

The negative impact of iodine deficiency on human health has prompted several actions, with iodization of salt being the most widely used approach ${ }^{3}$. While seaweeds represent a good source of iodine, fruits and vegetables contain very low iodine amounts, and several attempts are being carried out to set-up protocols for crop iodine biofortification ${ }^{4,5}$. Regretfully, the knowledge on the physiology of iodine in plants is almost non-existing. Iodine does not play any role in the physiology of plants, although this element can be taken-up by plants from the soil. The most obvious biotechnological approach to increase iodine content in plants would be to enhance its uptake. In this work we demonstrated that indeed a higher iodine uptake can be achieved, by the overexpression of the human NIS. Interestingly, we found that, unless $H O L-1$ was knocked-out, the increased iodine uptake driven by NIS was not accompanied by iodine accumulation in the plant. In NIS plant, the higher uptake rate of iodine further increased the already high $H O L-1$ expression (Fig. S2; Fig. 2f) vanishing the effect of NIS in terms of net iodine accumulation. Sodium, which is co-transported with iodine by NIS, is instead retained in the plant (Fig. 1g). The relevance of iodine volatilization as methyl iodide in plants exogenously fed with iodine suggests that iodine biofortification programmes of crops such as rice would result in the release of massive amounts of methyl iodide, a threat for the ozone layer ${ }^{13,14}$. Rice is indeed the best candidate for iodine biofortification programmes, as this cereal represents the staple food for millions of people in areas where iodine deficiency is a plague. Nowadays, five percent of methyl iodide arise from rice fields worldwide ${ }^{12}$, and these values would dramatically increase should rice iodine biofortification programs be put in place without a careful genetic evaluation of rice genotypes for low HOL activity.

In a perspective of crop biofortification with iodine, the removal of the volatilization process rather than the increase of the iodine uptake would more markedly affect the final iodine content in plants. Intelligent breeding for crops with low HOL activity would not only be important in iodine biofortification efforts but would also reduce the release of the ozone layer-threatening methyl halides into the atmosphere ${ }^{13,14}$.

\section{Methods}

Plant material and growth conditions. Arabidopsis thaliana, Columbia-0 (Col-0) and glabral ( $g l 1$ ) backgrounds were used in the experiments. After germination, plants were transplanted into soil or transferred into a hydroponic system, according to the kind of experiment. Plants grown in soil (Hawita Flor, Germany) were watered twice a week. The hydroponic system was based on thick gravel (3-5 $\mathrm{mm}$ in diameter) and a nutrient solution, whose composition was as follows (concentrations expressed in $\mathrm{mM}$ ): $\mathrm{Ca}\left(\mathrm{NO}_{3}\right)_{2} 3.5, \mathrm{KNO}_{3} 1.5, \mathrm{KH}_{2} \mathrm{PO}_{4} 0.5, \mathrm{MgSO}_{4} 0.75$, (concentrations expressed in $\mu \mathrm{M}$ ): Fe-Na-EDTA 72, $\mathrm{H}_{3} \mathrm{BO}_{3} 52, \mathrm{MnCl}_{2} 10, \mathrm{ZnSO}_{4} 2, \mathrm{CuCl}_{2}$ 1.6, $\left(\mathrm{NH}_{4}\right)_{6} \mathrm{MO}_{7} \mathrm{O}_{24}$ 0.08. Fresh solution was added weekly. All plants were grown in plastic pots (diameter $5 \mathrm{~cm}$ ) in a growth chamber, with $80 \mu \mathrm{mol} \mathrm{m}^{-2} \mathrm{~s}^{-1}$ PAR (GroLux, OH, USA), $12 \mathrm{~h}$ light photoperiod, $23^{\circ} \mathrm{C}$ temperature, $55 \%$ relative humidity.

The analysis of iodine toxicity was performed using plants grown in pots. When plants were 2 weeks old, they were fed $\mathrm{NaI}$ twice a week for four weeks with a $\mathrm{NaI}$ solution ( $5 \mathrm{ml} /$ pot at the concentrations shown in figure).

For the analysis of NIS gene expression, NIS seedlings were grown for two weeks in vertical plates containing $0.5 \mathrm{X}$ MS medium, $1 \%$ sucrose and $0.9 \%$ agar in a growth chamber (same conditions as described above). After two weeks, some of the plates were transferred for $2 \mathrm{~h}$ at $30^{\circ} \mathrm{C}$ or $37^{\circ} \mathrm{C}$. Plantlets were collected from all the treated plates as well as from control untreated plates (remained at $23^{\circ} \mathrm{C}$ ).

Col-0 hol-1 mutant line (T-DNA.SALK_005204) ${ }^{21}$, with a T-DNA insertion in At2g43910, was obtained from the European Arabidopsis Stock Centre (NASC, University of Nottingham, UK). Homozygous plants were identified via PCR screening of genomic DNA using gene-specific primers (listed in Supplementary Table 1) together with T-DNA left border-specific primers.

NISxhol-1 plants were obtained by crossing NIS plants from two independent lines (line 16 and 17) with hol-1 mutants and then screening the resulting F2 generations by PCR.

For the non-radioactive iodide uptake and the induction of the HOL-1 gene, 4week-old plants grown in soil, were treated with various quantities of potassium iodide (KI) (as specified in the figure legends). KI at different concentrations was distributed in the soil as a single treatment dissolved in $35 \mathrm{ml}$ deionized water, to give the final quantities indicated in the text and in the figure legends. The experiments were carried out for eight days, with samples taken at different time-points. Three replicates were analyzed for each treatment and/or time-point. Experiments of nonradioactive iodide uptake were also repeated with $\mathrm{NaI}$ and with plants grown both in soil and in a hydroponic system.

For liquid cultures, sterilized seeds were plated in multiwell plates containing $0.5 \mathrm{X}$ MS medium, $0.5 \%$ sucrose and 0.5X PPM in a final volume of $2 \mathrm{ml}$. Seeds were grown under continuous light and shaken at a temperature of $23^{\circ} \mathrm{C}$ for two weeks. Treatment with iodide was carried out by placing the seedlings in a fresh MS liquid medium 
supplied with 5 and $25 \mathrm{mM} \mathrm{KI}$. Samples were taken after 0 and $20 \mathrm{~min}$. Three biological replicates were analyzed.

Preparation of constructs and transgenic plants. The whole coding sequence of human NIS (hNIS) with KpnI and BamHI restriction sites at 5' and $3^{\prime}$ end, respectively, was obtained by PCR from the plasmid pcDNA3-Hnis ${ }^{22}$. The amplified $h N I S$ was inserted into a genetic cassette downstream of a Cauliflower mosaic virus 35 S promoter and upstream of a Nopaline synthase (NOS) terminator using its KpnI and BamHI ends. For the plant transformation, a pBIN- $h N I S$ binary vector was used. pBIN-hNIS is a pBIN19-derived plasmid carrying the 35S promoter:: $h$ NIS::NOS terminator genetic cassette inserted between the EcoRI and ClaI restriction sites. The plasmid was introduced into the Agrobacterium tumefaciens GV3101 strain, using the freeze-thaw method.

gll background of Arabidopsis was used for plant transformation. Transgenic plants were obtained using the floral dip method $^{23}$. T0 seeds were screened for kanamycin resistance (pBIN-hNIS contains the NptII gene coding for neomycin phosphotransferase II under the control of the pNOS promoter, thus conferring kanamycin resistance to plants). Homozygous lines were identified in T2 populations by analyzing segregation ratios. T3 or subsequent generations of line 16 and 17 (not shown), were used in the other experiments. Lines 16 and 17 carried independent insertions, as resulted by TAIL-PCR amplification ${ }^{24}$ of T-DNA flanking sequences (Figs. S3 and S4). Primers used for TAIL-PCR analysis are listed in Supplementary Table 1.

Quantitative RT-PCR analyses. Total RNA was extracted from the plant material (seedlings or rosette leaves, as indicated in the figure legends) using the RNeasy kit (Qiagen, CA, USA) in accordance with the manufacturer's instructions. Following a DNase treatment using the TURBO DNA-free kit (Ambion, TX, USA), $5 \mu \mathrm{g}$ of RNA were reverse transcribed into cDNA using the Superscript III reverse transcriptase kit (Invitrogen). Quantitative RT-PCR amplifications were carried out with the ABI Prism 7300 Sequence Detection System (Applied Biosystems, Life Technologies, CA, USA), using a TaqMan Universal PCR Master Mix (Applied Biosystems) for the amplification of $h N I S$ and a Power SybrGreen Master Mix (Applied Biosystems) for the amplification of HOL-1, HSP25.3 (At4g27670) and UBQ10 (At4g05320). UBQ10 was used as a reference gene. Primers are listed in Supplementary Table 1. The relative quantification of gene expression was performed using the comparative threshold cycle method, as described in the ABI PRISM 7700 Sequence Detection System User Bulletin Number 2 (Applied Biosystems).

Radioactive iodine uptake experiments. Arabidopsis plants, grown in hydroponics, were treated with radioactive iodine $\left({ }^{125} \mathrm{I}\right.$ as $\left.\mathrm{NaI}\right)$ starting at the age of four weeks (rosette stage) or six weeks (flowering stage) after germination. Each iodine treatment consisted in giving $1 \mathrm{ml}$ of a $0.25 \mu \mathrm{Ci} \mathrm{ml}{ }^{-1} \mathrm{Na}^{125} \mathrm{I}$ solution (approximately $0.1 \mathrm{pmol}$ $\mathrm{Na}^{125} \mathrm{I}$ ), directly added to the hydroponic medium. A total of three iodine feeding treatments were performed in each experiment, at two-day intervals, for a total amount of 0.3 pmol iodide supplied per plant. Plants during the iodine treatment (approx. a week) were grown at a temperature of $23^{\circ} \mathrm{C}$ or $30^{\circ} \mathrm{C}$ as indicated in the figure legends. The two different temperatures did not result in any differential effect on the plant growth, due to the short period of time. Each experiment was replicated three times.

Nitrate experiment. Arabidopsis plants grown in hydroponics were divided into two groups, starting from the beginning of the iodine treatment. One set of plants was maintained in the usual complete hydroponic solution (containing $5 \mathrm{mM}$ nitrate), while another identical set was moved to a modified nutrient solution in which the nitrate content was diluted to a final concentration of $0.5 \mathrm{mM}$ nitrate. As described above, three iodine feeding treatments $\left(1 \mathrm{ml}\right.$ for each treatment of a $0.25 \mu \mathrm{Ci} \mathrm{ml}^{-1}$ $\mathrm{Na}^{125}$ I solution, approx. $0.1 \mathrm{pmol}$ ) were carried out, at two-day intervals, for a total amount of iodine supplied per plant equal to $0.3 \mathrm{pmol}$. The two different nitrate levels did not produce any differential effect on the plant growth, due to the short period of time. Each experiment was replicated three times.

Visualization of radioactive iodine uptake. At the end of each uptake experiment plants were cut at the hypocotyl level, gently washed, blotted onto filter paper, and then exposed for two days to a Multipurpose Phosphor Storage Screen (Packard, CT, USA) in order to obtain a digital image of the radioactivity distribution. Digital images were obtained and analyzed using a Cyclone Storage Phosphor System (Packard). Data as DLUs (Digital Light Units) were expressed as relative radioactivity levels $(100=$ wild type) and the scanned images are shown using false colors, where red and blue indicate a high or low level of radioactivity, respectively.

Iodine, sodium and nitrogen measurements. The iodine content of Arabidopsis plants (rosette leaves) was measured using the Inductively Coupled Plasma - Mass Spectrometry (ICP-MS) technique ${ }^{25}$. Iodine was determined using an isotope dilution with iodine-127. The iodine concentration in the samples was evaluated using a calibration curve obtained with the standard additions method. The sodium content was measured by the Inductively Coupled Plasma by Optical Emission Spectrometry (ICP-OES) technique ${ }^{26}$. Total nitrogen was measured using the Kjeldahl method ${ }^{27}$ and expressed as grams per $100 \mathrm{~g}$ of fresh weight. Three replicates were analyzed for each treatment. Analyses were carried out by Neotron Spa (Italy).
Analysis of HOL-1 expression in publicly available microarray datasets. The average mRNA abundance of HOL-1 (At2g43910) was measured in various tissues and at different developmental stages using Affymetrix ATH1:22K publicly available microarray datasets. Data were retrieved from the Genevestigator ${ }^{28}$ webtool on January 18th, 2012.

1. Dohan, O. et al. The sodium/iodide symporter (NIS): characterization, regulation, and medical significance. Endocrine Rev. 24, 48-77 (2003).

2. Winger, R. J., König, J. \& House, D. A. Technological issues associated with iodine fortification of foods. Trends Food Sci. Tech. 19, 94-101 (2008).

3. Delange, F. \& Lecomte, P. Iodine supplementation: benefits outweigh risks. Drug Saf. 22, 89-95 (2000).

4. Dai, J. L., Zhu, Y.-G., Zhang, M. \& Huang, Y.-Z. Selecting iodine-enriched vegetables and the residual effect of iodate application to soil. Biol. Trace Elem. Res. 101, 265-276 (2004).

5. Weng, H. X. et al. Biogeochemical transfer and dynamics of iodine in a soil-plant system. Environ. Geochem. Health 31, 401-411 (2009).

6. Zhu, Y.-G., Huang, Y.-Z., Hu, Y. \& Liu, Y.-X. Iodine uptake by spinach (Spinacia oleracea L.) plants grown in solution culture: effects of iodine species and solution concentrations. Environ. Int. 29, 33-37 (2003).

7. White, P. J. \& Broadley, M. R. Biofortification of crops with seven mineral elements often lacking in human diets - iron, zinc, copper, calcium, magnesium, selenium and iodine. New Phytol. 182, 49-84 (2009).

8. Weng, H. X. et al. Mechanism of iodine uptake by cabbage: effects of iodine species and where it is stored. Biol. Trace Elem. Res. 125, 59-71 (2008).

9. Herrett, R. A., Hatfield, H. H. Jr., Crosby, D. G. \& Vlitos, A. J. Leaf abscission induced by the iodide ion. Plant Physiol. 37, 358-363 (1962).

10. Mackowiak, C. L. \& Grossl, P. R. Iodate and iodide effects on iodine uptake and partitioning in rice (Oryza sativa L.) grown in solution culture. Plant Soil 212, 135-143 (1999)

11. Landini, M., Gonzali, S. \& Perata, P. Iodine biofortification in tomato. J. Plant Nutr. Soil Sci. 174, 480-486 (2011).

12. Redeker, K. R. et al. Emissions of methyl halides and methane from rice paddies. Science 290, 966-969 (2000).

13. Rhew, R. C, Østergaard, L., Saltzman, E. S. \& Yanofsky, M. F. Genetic control of methyl halide production in Arabidopsis. Curr. Biol. 13, 1809-1813 (2003).

14. Nagatoshi, Y. \& Nakamura, T. Characterization of three halide methyltransferases in Arabidopsis thaliana. Plant Biotechnol. 24, 503-506 (2007).

15. Itoh, N. et al. Involvement of S-adenosylmethionine-dependent halide/thiol methyltransferase (HTMT) in methyl halide emissions from agricultural plants: isolation and characterization of an HTMT-coding gene from Raphanus sativus (daikon radish). BMC Plant Biol. 9, 116 (2009).

16. Kinney, A. J. Metabolic engineering in plants for human health and nutrition. Curr. Opin. Biotechnol. 17, 130-138 (2006).

17. Storozhenko, S. et al. Folate fortification of rice by metabolic engineering. Nat. Biotechnol. 25, 1277-1279 (2007).

18. Wirth, J. et al. Rice endosperm iron biofortification by targeted and synergistic action of nicotianamine synthase and ferritin. Plant Biotech. J. 7, 631-644 (2009).

19. Tonacchera, M. et al. Relative potencies and additivity of perchlorate, thiocyanate, nitrate, and iodide on the inhibition of radioactive iodide uptake by the human sodium iodide symporter. Thyroid 14, 1012-1019 (2004).

20. Mynett, A. \& Wain, R. L. Herbicidal action of iodide: effect on chlorophyll content and photosynthesis in dwarf Phaseolus vulgaris. Weed Res. 13, 101-109 (1973).

21. Alonso, J. M. et al. Genome-wide insertional mutagenesis of Arabidopsis thaliana. Science 301, 653-657 (2003).

22. Tonacchera, M. et al. Congenital hypothyroidism due to a new deletion in the sodium/iodide symporter protein. Clin. Endocrinol. 59, 500-506 (2003).

23. Clough, S. J. \& Bent, A. F. Floral dip: a simplified method for Agrobacteriummediated transformation of Arabidopsis thaliana. Plant J. 16, 735-743 (1998).

24. Liu, Y.-G., Mitsukawa, N., Oosumi, T. \& Whittier, R. F. Efficient isolation and mapping of Arabidopsis thaliana T-DNA insert junctions by thermal asymmetric interlaced PCR. Plant J. 8, 457-463 (1995).

25. Yoshida, S., Muramatsu, Y., Katou, S. \& Sekimoto, H. Determination of the chemical forms of iodine with IC-ICP-MS and its application to environmental samples. J. Radioanal. Nuclear Chem. 273, 211-214 (2007).

26. Alberta Environment Methods Manual, Pollution Control Laboratory, Edmonton, Canada (1979).

27. ISO 937-1978 (E). Meat and meat products -- Determination of nitrogen content.

28. Laule, O., Szabo, G., Wessendorp, F., Bleuler, S., Oertle, L., Widmayer, P. Gruissem, W. \& Zimmermann, P. Genevestigator V3: a reference expression database for the meta-analysis of transcriptomes. Adv. Bioinformatics, 420747 (2008).

\section{Author contributions}

M.L., experimental design, analysis of transgenic lines, expression analysis, uptake experiments; S.G., experimental design, molecular cloning, Arabidopsis transformation; C.K., analysis of transgenic lines, uptake experiments; P.A. and A.D., NIS cloning; A.A., P.V, 
and A.P., general discussion, laboratory coordination; M.T. and P.P., experimental design, initiation and coordination of the research project. P.P. and S.G. wrote the manuscript.

\section{Additional information}

Supplementary information accompanies this paper at http://www.nature.com/ scientificreports
Competing financial interests: The authors declare no competing financial interests. License: This work is licensed under a Creative Commons Attribution-NonCommercial-ShareAlike 3.0 Unported License. To view a copy of this license, visit http://creativecommons.org/licenses/by-nc-sa/3.0/

How to cite this article: Landini, M. et al. Metabolic engineering of the iodine content in Arabidopsis. Sci. Rep. 2, 338; DOI:10.1038/srep00338 (2012). 